\title{
Validation of Dynamic Simulation Models on Uncertainty
}

\author{
Xiaojun Guo* and Shaojing Su \\ College of Mechatronics Engineering and Automation, National University of Defense Technology, Changsha, China \\ ${ }^{*}$ Corresponding author
}

\begin{abstract}
Autonomous underwater vehicles (AUV) play an important role in human activities. They are different from of the vehicles other areas (such as air, ground). Movement, communication, target detection all involve complex hydrology and climatic condition. For special application fields it's even more complex. Due to the limitation of experimental conditions, simulation tests are adopted to assist with the increase in the sample size. The credibility of the simulation models need to be evaluated, especially for dynamic models such as control systems. This paper studies the application of several common dynamic model validation methods in the AUV motion simulation model and compares the resolution of the model. It helps much if cluster validation and cross-validate of the evaluation results are adopted.
\end{abstract}

Keywords-autonomous underwater vehicles; validation; credibility; motion model

\section{INTRODUCTION}

With in-depth exploration and research of the field of marine science, autonomous underwater vehicles (AUV) play an important role in human activities. They are different from of the vehicles other areas (such as air, ground). Movement, communication, target detection all involve complex hydrology and climatic condition [1-3]. For special application fields it's even more complex. Due to the limitation of experimental conditions, AUV can not achieve the large sample size (hundreds or even thousands) of the test sample in the evaluation of the simulation model or in the field test. The simulation test can provide some auxiliary samples for the field test, but at the same time the availability of test data relates to the level of confidence of the simulation models [45].

There are two kinds of research to solve the problem of small sample test capacity: 1 . Simulation data is generated by simulation modeling; 2. Bayesian method is used to analyze small sample test data. The former utilizes the reproducibility of the simulation test to enrich the sample capacity, but its model credibility evaluation method needs to be researched according to the characteristics of the simulation object, and the availability of the simulation test data is determined by the confidence level. The latter is based on the likelihood function, and as far as possible to ensure that the decision-making risk is as small as possible using all possible information (overall information, sample information and a priori information) in order to achieve higher parameter estimation accuracy and narrower confidence interval in presence of the same or less sample size.

The credibility of the model is uncertain when compared with the credibility evaluation of deterministic models or the randomness of models, which may have one or more uncertainties. The uncertainty of system error and the uncertainty of inaccurate observation lead to the uncertainty of all the test or measurement data. Especially in credibility evaluation of the dynamic model, model validation results only focusing on an uncertainty are not universal. Some of the literature in the credibility of the performance characterized it in the absolute parameters, and study its nature, credibility is also part of a fuzzy or grey number concept. Different model validation methods use different evaluation criteria as a measure of credibility. While the credibility evaluation between the single model and the reference model is insignificant. A set of more than 2 credibility evaluations between the model to be evaluated and the reference model is more valuable. So the cluster test for reliability of the simulation models reflect even more information of the credibility of the assessment method of the degree of rationality and resolution.

\section{VALIDATION DATA}

The method of model validation for dynamic models is generally divided into time domain and frequency domain. In the time domain, there are the Theil's inequality coefficient (TIC), gray relational analysis, auto-correlation function and so on. In the frequency domain, there are maximum entropy spectral estimation method, window spectrum estimation method, wavelet analysis [6-7].

The validation in this paper is based on CLIMB data classification standard. According to CLIMB criteria, the data are divided into five levels, and high-level data can be used as a validation reference for low-level data. In this paper, L-2 (simulation data) is primarily validated using L-5 data (field data).

The field test roll data under the same test conditions of the AUV control system were selected to validate a set of three all-simulation data. The time series of the selected test data is primarily shown in the Figure 1 . The selected data is the rolling time series of 50s. The data of the simulation models 1 3 come from different simulations, and the field model data come from field test. The aim is to find the simulation model 
closest to the ideal model (reference model, CLIMB L-5) in a set of three simulation models.

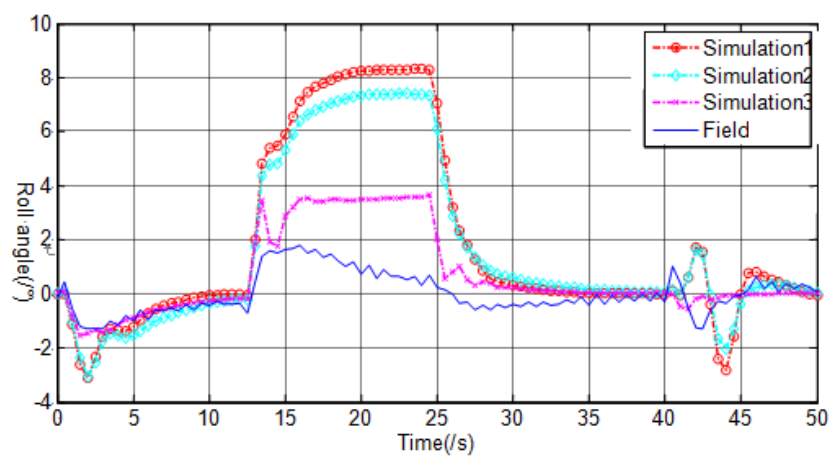

FIGURE I. DATA FOR VALIDATION

\section{VALIDATION METHODS AND CREDIBILITY EVALUATION}

\section{A. Maximum Entropy Spectral Estimation}

The stationary of the time series used in model validation is examined, and the field test data is used as the reference for non-stationary data segmentation [8].

After the stationary test and the stationary preconditioning, the first-order difference samples are tested for segmented stationary. According to W. Gersch and T. Brotherton's Akaike information criterion (AIC), there are two sections of AIC and non-segmented AIC, respectively, as shown in Figure 2. It can be seen in all sub-points, there are AIC $(1,2)>$ AIC (3), it is assumed that the first-order difference sample should be treated as a non-piecewise global stationary process.

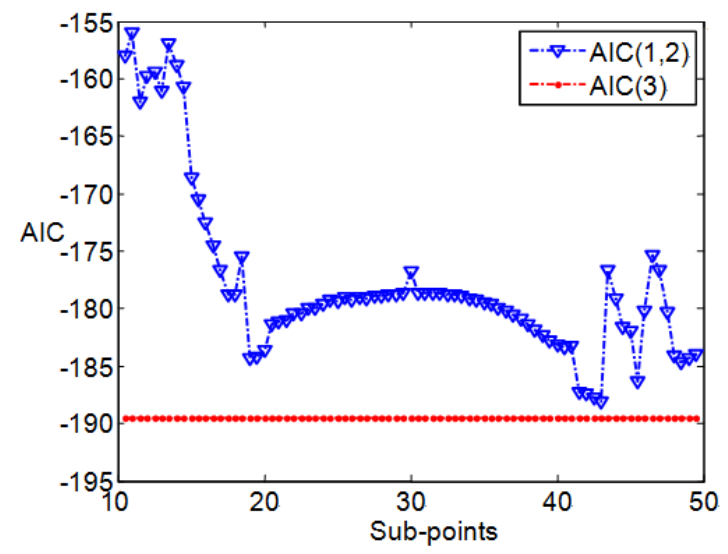

FIGURE II. AIC CHECK

As shown in Figure 3, it's statistic D value for the confidence level of 0.95 using maximum entropy spectrum estimation. It can be seen from the figure that, in the middle and low frequency region, the simulation model is better through the test, and the order of similarity between the simulation models and field model: simulation model 3> simulation model $2>$ simulation model 1 . And the percentage of the frequency points passing the test is $75.45 \%, 55.64 \%$ and $44.75 \%$, respectively.

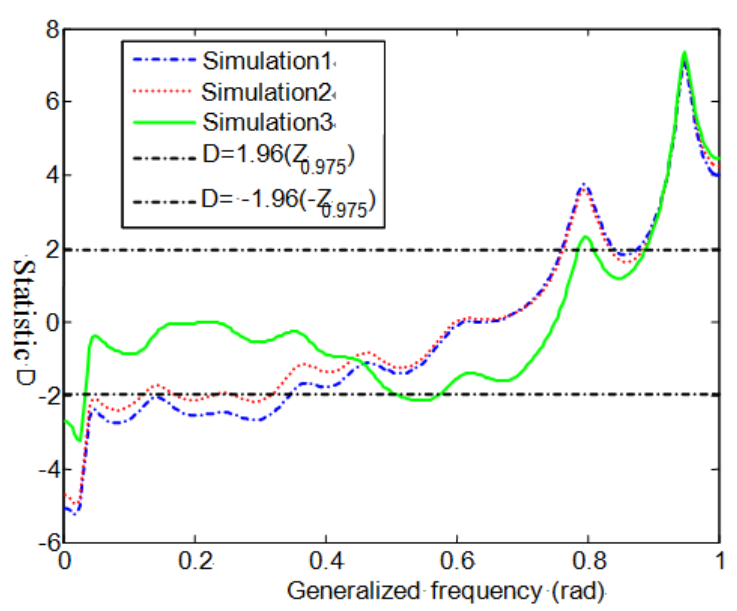

FIGURE III. STATISTIC D CHECK

\section{B. Wavelet Analysis}

The method of wavelet analysis can be used to validate the model. It is not necessary to test the data stationary, and do not need to fit the model. The field test data are decomposed into high and low frequency based on Daubechies wavelet, as shown in Figure 4[9].

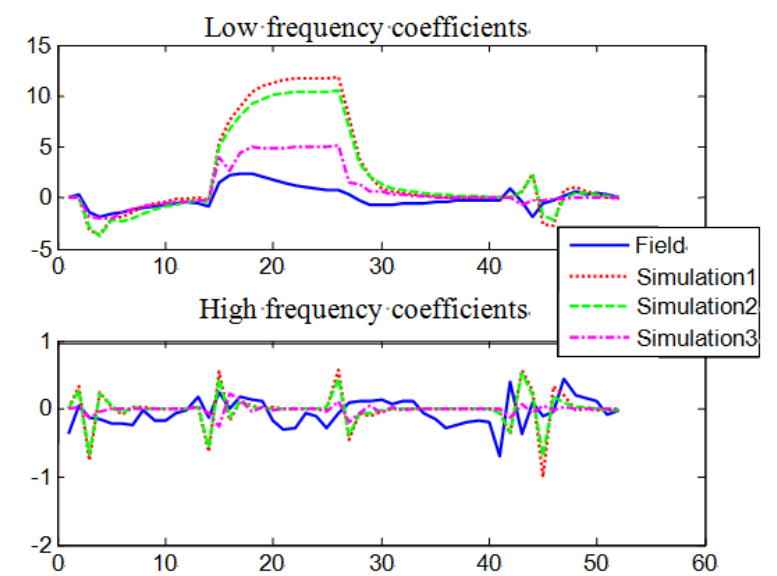

FIGURE IV. LOW AND HIGH FREQUENCY COEFFICIENTS VIA WAVELET ANALYSIS

The validation of the low-frequency part is taken using the maximum entropy spectral estimation method described above, and the distribution of the test statistic D and the accepted domain at the confidence level of 0.95 can be obtained. The percentages of the first-order low-frequency coefficients of simulation models 1-3 passing the statistic D check are $94.55 \%, 96.50 \%$ and $97.28 \%$ respectively, and the percentages of the second-order low-frequency coefficients passing the statistic D check are $91.83 \% 93.00 \%$ and $94.55 \%$ respectively. The frequency percentage of the third-order low-frequency coefficients passing the statistic D check are $82.49 \%$, $85.60 \%$ and $89.11 \%$ respectively.

Three orders high-frequency coefficients of the statistic D check shown in Table 1, showing that all three orders highfrequency coefficients have passed the test. 
TABLE I. HIGH-FREQUENCY COEFFICIENTS OF THE STATISTIC D CHECK

\begin{tabular}{|c|c|c|c|c|c|c|}
\hline & Simul.1 & Ratio & Simul.2 & Ratio & Simul.3 & Ratio \\
\hline First-order $T$ & 5.8554 & \multirow[t]{2}{*}{4.0107} & 4.6737 & \multirow[t]{2}{*}{4.149} & 2.7323 & \multirow[t]{2}{*}{4.1052} \\
\hline Threshold & 23.484 & & 18.655 & & 10.788 & \\
\hline Second-order $T$ & 6.7452 & \multirow[t]{2}{*}{3.9915} & 3.6567 & \multirow[t]{2}{*}{4.1508} & 6.0175 & \multirow[t]{2}{*}{4.1612} \\
\hline Threshold & 27.986 & & 15.179 & & 24.924 & \\
\hline Third-order $T$ & 28.212 & \multirow[t]{2}{*}{3.9192} & 22.818 & \multirow[t]{2}{*}{4.1418} & 3.7406 & \multirow[t]{2}{*}{4.255} \\
\hline Threshold & 115.81 & & 94.95 & & 15.919 & \\
\hline
\end{tabular}

\section{Grey Correlation Analysis}

The grey relational coefficient series obtained in (1)[10], (2)[11], (3)[12] and (4)[13-14] are shown in Figure 5, where the data to be validated is aligned with the field test data. It can be seen that various algorithms support the following conclusions when verifying the correlation between the simulation models and the field model data: simulation model slight

$3 \succ$ simulation model $2 \succ$ simulation model 1 , and the simulation model 3 has a better performance than the other two simulation models.

$\rho\left(a_{i j}, a_{i k}\right)=\frac{\min _{i} \min _{j} \min _{k, k \neq i}\left|a_{i j}-a_{i k}\right|+\zeta \max _{i} \max _{j} \max _{k, k \neq j}\left|a_{i j}-a_{i k}\right|}{\left|a_{i j}-a_{i k}\right|+\zeta \max _{i} \max _{j} \max _{k, k \neq j}\left|a_{i j}-a_{i k}\right|}$

$\rho\left(a_{i j}, a_{i k}\right)=\left(\frac{\min _{i} \min _{j} \min _{k, k j}\left|a_{i j}-a_{i k}\right|+\zeta \max _{i} \max _{j} \max _{k, k \neq}\left|a_{i j}-a_{i k}\right|}{\left|a_{i j}-a_{i k}\right|+\zeta \max _{i} \max _{j} \max _{k, k \neq j}\left|a_{i j}-a_{i k}\right|} \times \exp \left(-\eta_{j k}(i)\right)\right)^{1 / 2}$
$\rho\left(a_{i j}, a_{i k}\right)=\frac{\min _{i} \min _{j} \min _{k, k \neq j}\left|a_{i j}-a_{i k}\right|+\zeta \max _{i} \max _{j} \max _{k, k \neq j}\left|a_{i j}-a_{i k}\right|}{\left|a_{i j}-a_{i k}\right|+\zeta \max _{i} \max _{j} \max _{k, k \neq j}\left|a_{i j}-a_{i k}\right|+\left(\sum_{j=1}^{n}\left|a_{i j}-a_{i k}\right|^{2}\right) / n}$

$$
\rho\left(a_{i j}, a_{i k}\right)=\frac{\min _{i} \min _{j} \min _{k, k \neq j}\left|d_{k j}(i)\right|+\zeta \max _{i} \max _{j} \max _{k, k \neq j}\left|d_{k j}(i)\right|}{\left|d_{k j}(i)\right|+\zeta \max _{i} \max _{j} \max _{k, k \neq j}\left|d_{k j}(i)\right|}
$$

\section{CONCLUSIONS}

In the validation of the simulation model and the reference model, the subjective or objective, qualitative or quantitative credibility evaluation method is derived from the specific purpose and background of the application. Three typical dynamic model validation methods are illustrated above: frequency domain, time domain, time-frequency mixing domain. Although the three methods described quantitatively or qualitatively give the results of model cluster validation or cluster ordering, there is little literature on how to evaluate the reliability, rationality or resolution of validation methods.
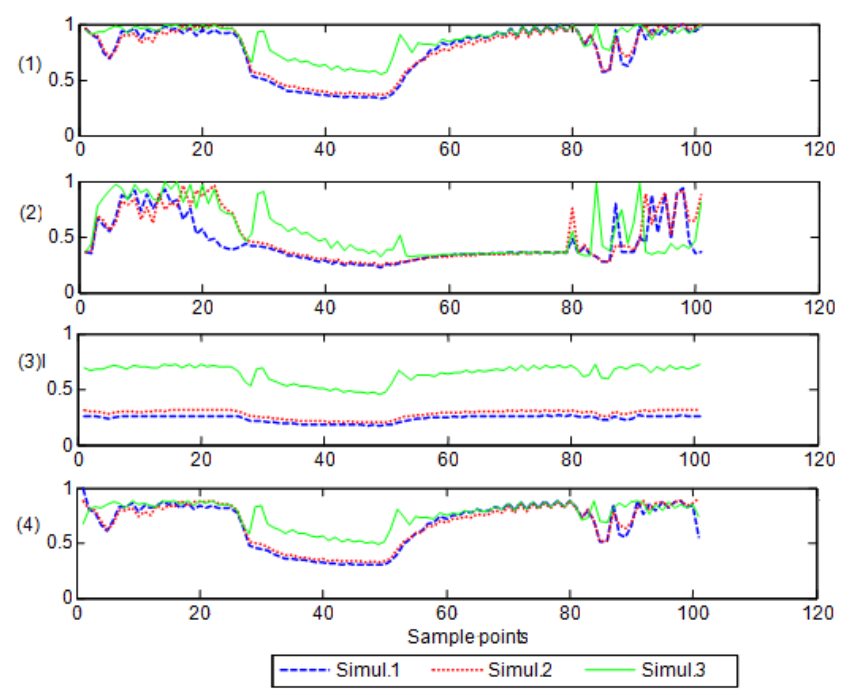

FIGURE V. GREY RELATIONAL COEFFICIENT SERIES

A simple validation result, especially the credibility of a single model to be tested and a reference model, is subjective or objective, and is only a measure based on the theoretical approach, but lacks a comprehensive reference standard. In this paper, a set of three models to be tested are selected to validate the model. When the model is validated by the maximum entropy spectrum estimation method, the frequency percentage of the three models passing the statistic D check is $44.75 \%, 55.64 \%, 75.45 \%$. From the test results it's easy to know, the models to be tested in the high frequency region have low pass rate. In wavelet analysis, after the decomposition of the high and low frequency, the low pass frequency coefficients of all the low-frequency coefficients of the frequency point test pass rate of more than $80 \%$. It also verifies the maximum entropy spectrum estimation method test result of high frequency part. GRA is based on the implicit information of the relational analysis. Different correlation measures also affect the results of the credibility evaluation.

In summary, the three methods support the consistency conclusion basically in the compatibility test with reference model (field model): simulation model $3>$ simulation model $2>$ simulation model 1 . In practical application, we need to use several methods according to different application objects, and use some means to cross-validate the evaluation results of each method.

\section{ACKNOWLEDGMENT}

The research is supported by Natural Science Foundation of China (No.61374008).

\section{REFERENCES}

[1] Xiuhua Shi,Xiaojuan Wang. Introduction to Underwater Weapons[M] Xian: Press of Northwestern Polytechnical University, 2005:32-38

[2] Xiaojuan Shi. Prime Torpedo Technology\{M]. Xian: Press of Northwestern Polytechnical University, 2005:42-47.

[3] Xingtang Liu, Bincheng liang, et al. Theory, Method and Technology for Complex Systems Modeling [M].Beijing:Science Press,2008:4. 
[4] Wen Zhao, Dewen Hu.Development of Validation of Weapon systemsVirtual Test. [J].Computer Mearement and Control,2008(1):1-5.

[5] Lee H. Virtual Test Track[J].IEEE TRANSACTIONS ON VEHICULAR TECHNOLOGY,2004(6):1818-1816.

[6] Ying Liao,Jiafang Deng, Jiahong Liang. M\&S and VV\&A [M].Changsha:Press of NUDT,2006:12-26.

[7] Yongcheng Sun, Yanqin Ma, Xianzhong Zhou,et al. Test and Evaluation ofValidation and Accreditation in Modeling and Simulatiuon. [J].Journal of System Simulation, 2005(17):2317-2320.

[8] Yi Zhang, Qingshan Jiang.. Efficacy Assessment of Early Warning Airplane based on Layered TOPSIS [J].System Engineering and Electrical Technology,2011,33(5):1051-1054.

[9] Hongyu Wang,Tianshuang Qiu, Zhe Chen. Analysis and Process of Non- stationary Rondom Signals [M].Beijng:Press of Defense Industry, 2008

[10] Spiegelhalter DJ, Best NG, Gilks WR, Inskip H. Markov chain Monte Carlo in practice[M]. London: Chapman \& Hall, 1996.

[11] Xiaoyan Wu, Lina Yang, Yanyan Zhou.Simulation Model Validation Based on Wavelet Analysis[J]. Journal of Air Force Engineering University (Natrual Science), 2008,9(6):52-57.

[12] Yongcheng Sun,Xianzhong Zhou,et al.Improvemnet of Simulation Model Validation Based on Grey Relational Analysis[J]. Journal of System Simulation,2005,17(3):522-525.

[13] Xiaojun Guo, Zhiping Huang, Chunwu Liu, Junpeng Hu. Solution for validation of simulation models using GRA after GRA. The Journal of Grey System, 2012, 2:119-132

[14] Xiaojun Guo, Zhiping Huang, Chunwu Liu. Validation of Torpedo Control System Simulation Models on Grey Relational Analysis. ICIECS2010 